F. Müller

I. Nitschke

\title{
Mundgesundheit, Zahnstatus und Ernährung im Alter
}

ausgeglichen werden. Gleichzeitig erschweren oft Mundtrockenheit und Dysphagie die Nahrungsaufnahme bei alten Menschen. Subjektiv oft nicht wahrgenommen stellen Prothesenträger ihre Nahrungsmittelauswahl um und greifen auf spezielle Zubereitungsarten zurück. Letztendlich zeigt der Zahnstatus einen Einfluss auf die Nahrungsaufnahme. Mit abnehmender Zahnzahl werden weniger Kalorien, Proteine, Fett, nichtstärkehaltige Polysacharide und Vitamine aufgenommen. Oft werden die fehlenden Kalorien durch vermehrte Zucker und Fettaufnahme ausgeglichen. Besonders zahnlose Personen mit geringer Schulbildung greifen zu einer eher fett- und zuckerhaltigen Ernährung. Auch nimmt der tägliche Verzehr von Früchten und Gemüse mit der Anzahl der Seitenzahnkontakte ab. Eine Verbesserung der Kaufunktion durch zahnärztliche Maßnahmen geht nicht automatisch mit einer verbesserten Nahrungsaufnahme einher und sollte daher immer mit einer Ernährungsberatung kombiniert werden.

Schlüsselwörter Zahnstatus Zahnverlust - Kaufunktion Mundgesundheit - Ernährung Speichel
Summary The loss of natural teeth impairs essentially the chewing function and can only partly be restored by the insertion of dental prostheses. Equally, xerostomia and dysphagia may aggravate the nutritional intake in older adults. Often denture wearers do subjectively not notice the adjustment of their food choice and the employment of special preparation techniques. Finally the dental state influences the nutritional intake. A reduced number of teeth correlates with the intake of less calories, proteins, fat, nonstarch polysaccharides and vitamins. Often missing calories are compensated by an increased consumption of sugar and fat. Especially edentulous persons with a low level of education choose a diet which is rich in fat and sugar. Further the daily intake of fruit and vegetables diminishes along with fewer occlusal contacts in posterior teeth. The restoration of the chewing function by dental intervention does not lead to an improvement of the nutritional intake by itself and should therefore always be complemented by nutritional advice.

Key words Dental state tooth loss - chewing function oral health- nutrition - saliva

E-Mail:

ina.nitschke@medizin.uni-leipzig.de 


\section{Einleitung}

Eine gesunde und ausgewogene Ernährung trägt wesentlich zum erfolgreichen Altern bei. Unter- und Mangelernährung sind jedoch nicht nur bei selbstständig lebenden sondern insbesondere bei institutionalisierten und hospitalisierten Senioren $\mathrm{zu}$ beobachten [5, 35]. Im Rahmen einer Genfer Studie enthielten $10 \%$ der Kühlschränke älterer Personen über 65 Jahre weniger als 3 Lebensmittel. Solch ein "leerer" Kühlschrank erhöhte die Wahrscheinlichkeit einer Hospitalisierung innerhalb der folgenden 30 Tage signifikant [2]. Die Beschaffung frischer Nahrungsmittel stellt für ältere Personen oft eine $\mathrm{He}$ rausforderung dar. Alters- und krankheitsbedingte Mobilitätsseinschränkungen erschweren den Weg zum Markt oder Supermarkt sowie den Transport schwerer Einkäufe. Ferner schränken nicht selten finanzielle Aspekte die Nahrungsmittelauswahl ein. Aber auch die Zubereitung einer warmen Mahlzeit ist im Alter nicht selbstverständlich. Ist das Leben durch Einsamkeit und Depressionen gekennzeichnet, fehlen oft der Appetit und die Motivation zum aufwendigen Kochen, der Griff zum „convenience food“ liegt nahe [35].

Ein weiterer Aspekt, der im Hinblick auf die Ursachen von Unter- und Mangelernährung oft unterschätzt und daher vernachlässigt wird, ist die Mundgesundheit [39]. Insbesondere der Zahnstatus, d.h. die Anzahl, Wertigkeit und Verteilung der Zähne, Karies, Parodontalerkrankungen, Zahnlockerungen sowie die Qualität und Art der prothetischen Versorgung bestimmen die Kaueffizienz und kann die Nahrungsmittelauswahl beeinflussen. Grundsätzlich bietet festsitzender Zahnersatz eine höhere Kaueffizienz als herausnehmbarer Zahnersatz, implantatretinierte
Prothesen sind dem konventionellen, rein schleimhautgetragenen Zahnersatz überlegen. Darüber hinaus können Xerostomie und Dysphagie die Nahrungsaufnahme beeinträchtigen. Das Beispiel der Karies verdeutlicht den circulus vitiosus von Kaufunktion, Ernährung und Zahnstatus (Abb. 1). Kariöse Zähne können zu Zahnverlust führen, welcher die Kaueffizienz verringert. Dieser führt wiederum zu einer veränderten Nahrungsauswahl, insbesondere der vermehrten Aufnahme von süßen und klebrigen Speisen, die weitere Karies und weiteren Zahnverlust verursachen. Mit dem Alter nimmt die Frequenz der Zahnarztbesuche ab (Abb. 2), so dass die Unterbrechung des circulus vitiosus durch zahnärztliche Intervention seltener stattfindet $[31,32]$. Da gleichzeitig die Frequenz der Arztbesuche ansteigt, sollte eine allgemeinärztliche Untersuchung die Inspektion der Mundhöhle einbeziehen um ggf. eine zahnärztliche Behandlung zu veranlassen.

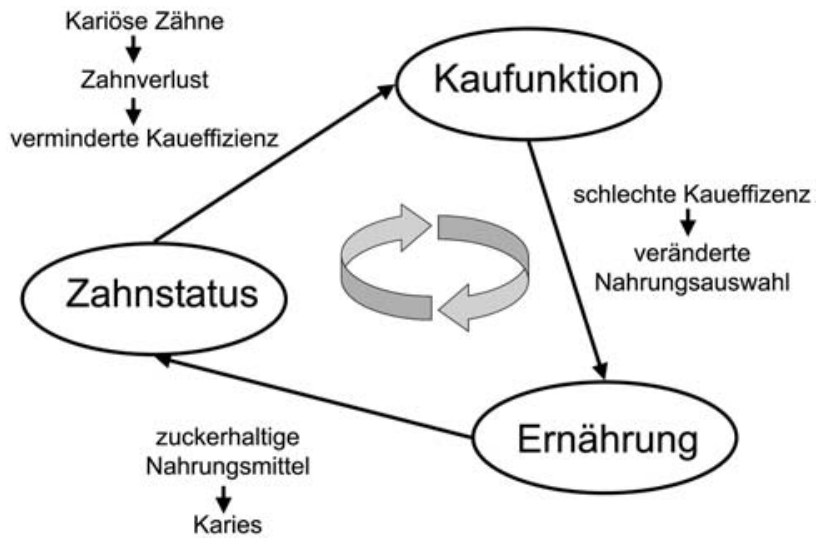

Abb. 1 Wechselwirkung zwischen Ernährung, Zahnstatus und Kaufunktion am Beispiel Karies
Abb. 2 Alter und Zeit seit dem letzten Zahnarztbesuch - Daten aus der Berliner Altersstudie [31]

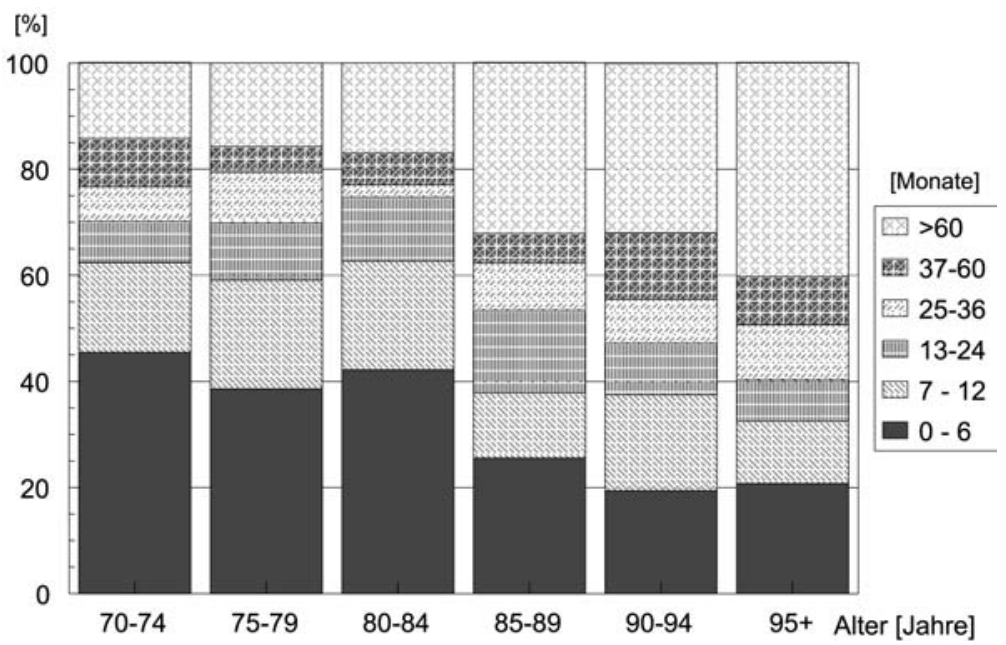




\section{Prävalenz von Zahnverlust und Zahnersatz}

Präventionskonzepte sowie Fortschritte in der restaurativen Zahnheilkunde haben dazu geführt, dass die natürlichen Zähne wesentlich länger erhalten bleiben. Bei gleichzeitig steigender Lebenserwartung wird herausnehmbarer Zahnersatz daher immer häufiger erst in einer späteren Lebensphase eingegliedert. Nichtsdestotrotz tragen laut der 3. Deutschen Mundgesundheitsstudie (DMS III) 24,8\% der 65- bis 74-Jährigen eine Totalprothese, d.h. sie sind zahnlos [20]. Mit zunehmendem Alter steigt dieser Anteil in der Bevölkerung, so konnte in der Berliner Altersstudie in der Kohorte der 85- bis 90-Jährigen einen Anteil von fast $80 \%$ Zahnlosen ermittelt werden [31]. Entsprechend ist ein Großteil der alten und sehr alten Bevölkerung bezüglich ihrer Kaufunktion eingeschränkt, da sie nur noch wenige oder gar keine natürlichen Zähne haben. Die prothetische Versorgung mit Hilfe von oralen Implantaten ist bis ins hohe Alter möglich, wird aber eher selten von betagten Menschen in Anspruch genommen.

\section{Kaueffizienz}

Der Begriff Kaueffizienz, auch als objektive Kauleistung beschrieben, bezeichnet die Fähigkeit, Nahrung innerhalb einer definierten Anzahl von Kauzyklen zu Partikeln unterschiedlicher Größe zu zerkleinern [22]. Klassische Testnahrung sind Erdnüsse oder Mandeln, die ein besonders günstiges Frakturverhalten zeigen. Aber auch künstliche Testnahrungen aus Silikon oder auf Gelatinebasis haben sich bewährt $[9,24]$. Nach dem Kauen wird der Speisebrei ausgespuckt und durch einen Stapel von Sieben mit abnehmender Maschengröße gespült. Durch Wiegen der einzelnen Siebe kann der Anteil, großer, mittlerer und sehr feiner Partikel bestimmt werden. Da sich beim Spülen auch Wasser in den Maschen verfängt, müssen die einzelnen Siebe vor dem Wiegen getrocknet werden. Die Siebmethode ist noch immer der "Goldstandard“ unter den Kaufunktionstests, jedoch ist sie aufwendig und bedarf des Satzes an Sieben (Abb. 3). Computergestützte Partikelanalysen erreichen die gleiche Präzision, sind jedoch noch teurer und aufwendiger [29]. Um Kaufunktionstests auch bei einer geriatrischen Routineuntersuchung durchführen zu können wurde der „Karottentest“ beschrieben, wobei der Grad der Pulverisierung der Karotte lediglich durch "Augenscheinnahme" bestimmt wird [41]. Nützlich und einfach $\mathrm{zu}$ handhaben sind hingegen Kaueffizienztests mit Kaugummi. Hier kann die Form des Bolus nach 20 Kauzyklen oder der Gewichtsverlust durch den in

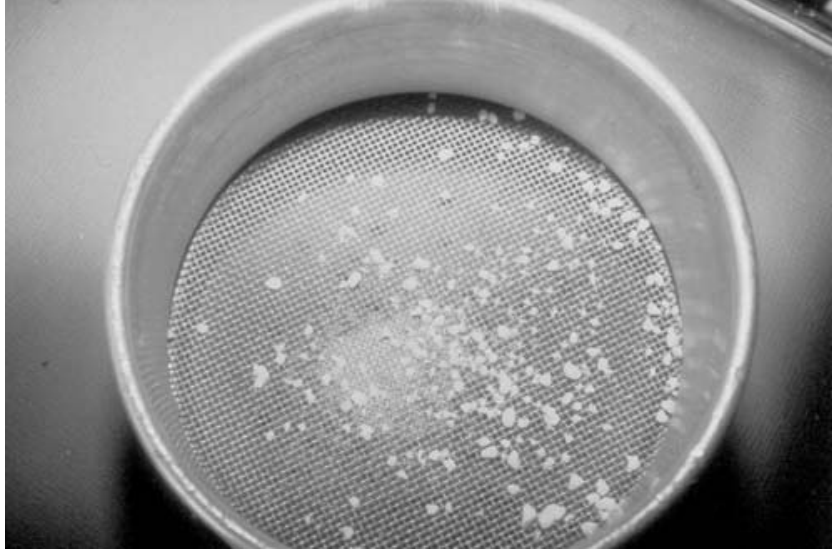

Abb. 3 Siebe mit absteigender Maschengröße zur Messung der Kaueffizienz

Lösung gegangenen Zuckergehalt beurteilt werden [14]. Bei zweifarbigem Kaugummi kann die Kaueffizienz zusätzlich aufgrund der Farbdurchmischung bestimmt werden [34].

\section{Kaueffizienz und Zahnstatus}

Der Verlust der Zähne geht mit einem signifikanten Verlust an Kaueffizienz einher, da eine geringere Zahnzahl mit entsprechend verminderter Anzahl von Kauflächen die Partikelzerkleinerung verringert [1, 11]. Obwohl Alterserscheinungen kaum einen Einfluss auf die Kaueffizienz haben, zeigt sich der Muskelquerschnitt der Kaumuskulatur im Alter signifikant verringert. Diese Atrophie wird durch den Verlust der Zähne weiter verstärkt [30]. Entsprechend nimmt die maximale Kieferschließkraft ab. Bei Trägern von herausnehmbarem Zahnersatz, der ganz oder teilweise schleimhautgelagert ist, wird die maximale Kieferschließkraft zusätzlich durch die Schmerzschwelle der prothesentragenden Lagergewebe begrenzt. Auch passgenauer Zahnersatz kann die Kaueffizienz daher nur teilweise wiederherstellen. Im Rahmen der "Dutch Academic Overdenture Study“ wird die Kaueffizienz mit unterschiedlichen Zahnstaten bzw. prothetischen Versorgungen verglichen [9] (Abb. 4). Hierzu wurde ein 5,6 mm großes Silikonkaugut mit 40 bzw. 60 Kauzyklen gekaut und dessen Zerkleinerung anschließend mittels der Siebmethode analysiert. Dabei zeigten die natürlich vollbezahnten Probanden unabhängig von deren Alter die höchste Kaueffizienz, gefolgt von den Probanden mit verkürzter Zahnreihe (fehlende Molaren) und denen, die mit einer Deckprothese (Prothese abgestützt auf natürlichen Wurzeln) versorgt waren. Die zahnlosen Probanden, die mit Totalprothesen kauten, zeigten die geringste Kaueffizienz, jedoch in Abhängigkeit 
Abb. 4 Zusammenhang von Zahnstatus, prothetischer Versorgung und Kaueffizienz, gemessen am Pulverisierungsgrad eines 5,6 mm Silikonblocks (nachgezeichnet nach [9])

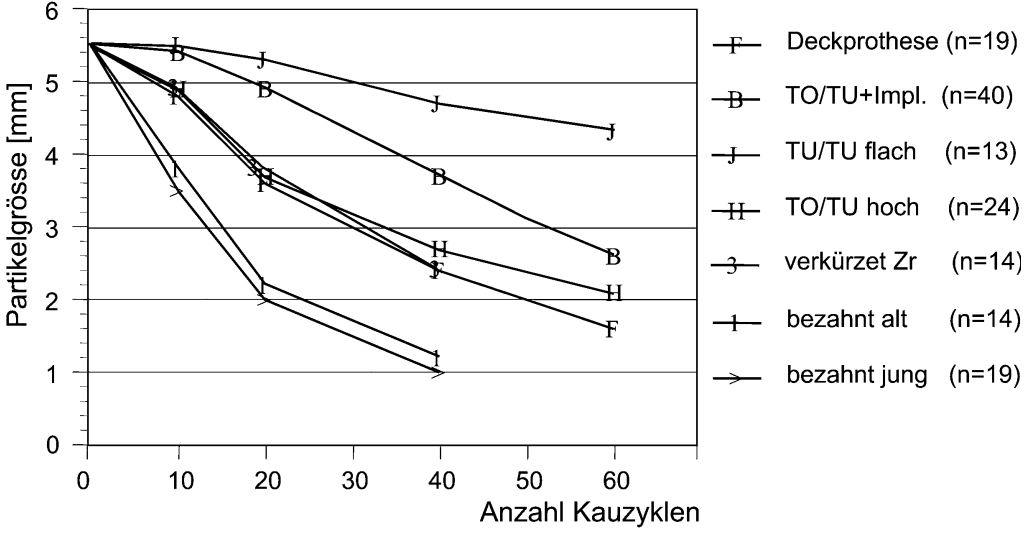

Gibt es Speisen, die Sie nur mit Schwierigkeiten essen

von der Atrophie der Kieferkämme große Unterschiede. Eine Implantatverankerung der Unterkiefertotalprothese konnte die Kaueffizienz verbessern, da davon auszugehen ist, dass sich lediglich Patienten mit einer starken Atrophie der Kieferkämme dem chirurgischen Eingriff unterzogen haben.

\section{Zahnstatus und Ernährung}

Die durch Zahnverlust verminderte Kaueffizienz bedingt nicht selten eine Einschränkung der Nahrungsmittelauswahl [13, 38]. Da der Funktionsverlust i.d.R. jedoch schleichend eintritt, wird dieser subjektiv kaum wahrgenommen. Auch unterschiedliche $\mathrm{Zu}$ bereitungsarten, wie z.B. das Dämpfen von Äpfeln, das Entfernen von Brotrinde oder das Mixen von Fleisch, werden zur Kompensation des Kaufunktionsverlustes herangezogen. Millwood und Heath konnten zeigen, dass Prothesenträger nur wenige Nahrungsmittel als „schwer zu kauen“ einschätzen [25] (Abb. 5). Dahingegen gab es mehrere Nahrungsmittel, die aufgrund dieser Schwierigkeiten einfach aus dem Nahrungsplan gestrichen wurden (Abb. 6). Dabei stellen für Prothesenträger nicht nur die extrem harten Nahrungsmittel eine Herausforderung dar, auch klebrige Speisen wie Brot können durch das Lösen der Prothese vom Lager Schwierigkeiten bereiten. Enthält eine Frucht oder Marmelade kleine Kerne, so können sich diese unter die Prothesenbasis setzen und extreme Schmerzen verursachen. Nicht zuletzt stellt Salat ein Problem dar, wenn die Blätter so fein sind, dass sie von den künstlichen Zähnen nur schwer durchteilt werden können.

Die veränderte Nahrungsmittelauswahl hat letztendlich auch Einfluss auf die Nahrungsaufnahme [19]. Dhaliwal beschrieb einen Zusammenhang zwischen der Anzahl der okklusalen Kontakte zwischen natürlichen Oberkiefer- und Unterkieferzähnen im Seitenzahnbereich und dem täglichen Verzehr von

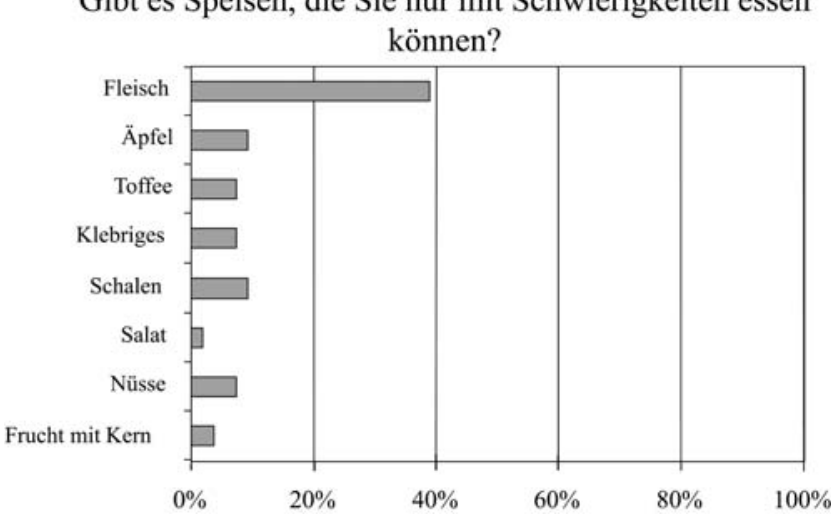

Abb. 5 Angaben zahnloser Patienten zu Nahrungsmitteln, die als schwierig zu essen empfunden werden [nach Millwood und Heath, 25]

Welche Lebensmittel lassen Sie weg, weil sie zu schwierig zu essen sind?

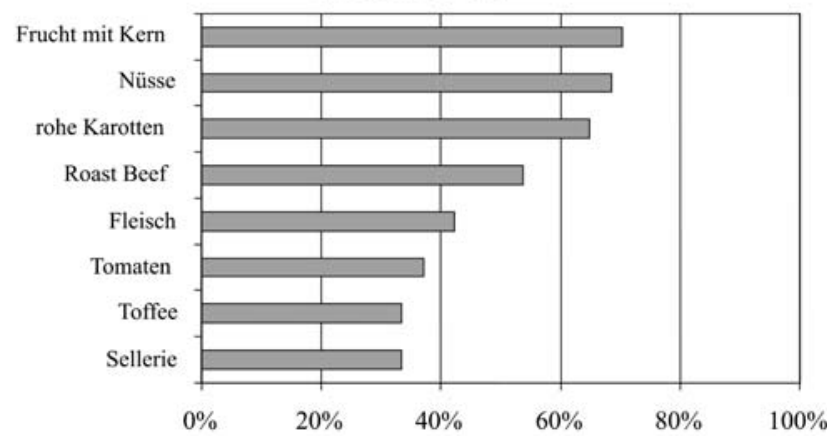

Abb. 6 Angaben zahnloser Patienten zu Nahrungsmitteln, die aufgrund mastikatorischer Schwierigkeiten nicht (mehr) gegessen werden [nach Millwood und Heath, 25]

Früchten und Gemüse [4]. Sheiham und Mitarbeiter konnten zeigen, dass mit abnehmender Zahnzahl weniger Kalorien, Proteine, Fett, nicht-stärkehaltige Polysaccharide und Vitamine aufgenommen wurden [37] (Tabelle 1). Andere Studien belegen, dass die 
Tab. 1 Nahrungsaufnahme und Zahnstatus - Ergebnisse einer Querschnittstudie von A Sheiham et al. [36]

\begin{tabular}{lrrr}
\hline & $\begin{array}{c}\text { Zahnlos } \\
\mathrm{n}=287\end{array}$ & $\begin{array}{c}1-10 \text { Zähne } \\
\mathrm{n}=131\end{array}$ & $\begin{array}{c}\text { 21+ Zähne } \\
\mathrm{n}=123\end{array}$ \\
\hline Energie (Kcal) & 1583 & 1700 & 1842 \\
Protein (g) & 60 & 66 & 71 \\
Fett (g) & 64 & 67 & 67 \\
Nicht-stärkehaltige & 11 & 13 & 16 \\
Polysaccharide (g) & & & \\
Calzium (mg) & 722 & 825 & 884 \\
Vitamin A ( $\mu \mathrm{g})$ & 1036 & 1374 & 1268 \\
Vitamin C (mg) & 60 & 82 & 83 \\
Niacin (mg) & 27 & 33 & 32 \\
Vitamin E (mg) & 8 & 10 & 12 \\
\hline
\end{tabular}

fehlenden Kalorien durch vermehrte Zucker und Fettaufnahme ausgeglichen werden [17]. Besonders zahnlose Personen mit geringer Schulbildung greifen $\mathrm{zu}$ einer eher fett- und zuckerhaltigen Ernährung [23].

\section{Mundtrockenheit}

Im Alter lässt die Speichelmenge, die durch Stimulation abgegeben werden kann, nach, dahingegen bleibt die Menge an Ruhespeichel, die zur Gesunderhaltung der Mundhöhle ausreicht, nahezu unverändert [31] (Abb. 7). Etwa jeder dritte alte Mensch leidet unter einer subjektiv empfundenen Mundtrockenheit, welche die Lebensqualität deutlich einschränkt $[15,21]$. Selten ist die Mundtrockenheit durch systemische Erkrankungen wie das Sjörgen-Syndrom oder Radiatio bedingt, meist ist die Ursache in den Nebenwirkungen von Medikamenten zu finden. Zur Formung eines Speisebolus sowie zum Schlucken ist eine ausreichende Menge Speichel unerlässlich. Weitere unangenehme Folgen der Xerostomie sind Sprachprobleme, häufige Entzündungen der Schleimhäute, Verlust an Prothesenretention sowie eine erhöhte Inzidenz von Karies und Abrasionen an den Zähnen. Tabelle 2 enthält Beobachtungen, die anamnestisch auf eine Mundtrockenheit hinweisen können [6]. Leider sind die therapeutischen Möglichkeiten für die Xerostomie sehr begrenzt. Das Kauen von Kaugummi kann den Speichelfluss über die Parodontalrezeptoren stimulieren, auch gustatorische Reize, beispielsweise das Lutschen von zuckerfreien Anisbonbons, regt den Speichelfluss an. Zitrone erhöht den Speichelfluss ebenfalls, greift aber gleichzeitig den Zahnschmelz an. Neben einer ausreichenden Flüssigkeitszufuhr beschränken sich viele Patienten auf regelmäßige kleine Schlucke Wasser und verzichten somit auf den Einsatz von künstlichem Speichel, der häufig als unangenehm empfunden wird.

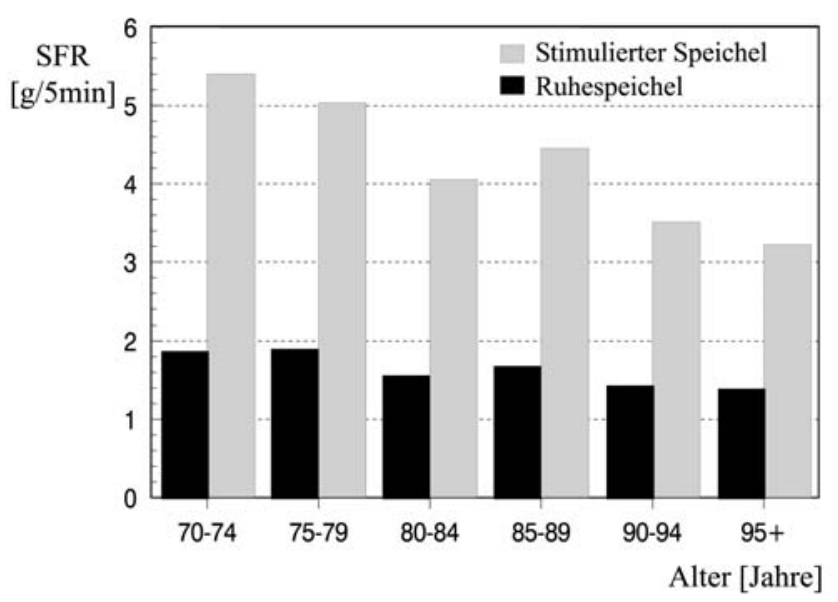

Abb. 7 Speichelfließrate (SFR) in Ruhe und nach 5 min Stimulation durch Kauen von Wachs [nach Nitschke et al. 31]

Tab. 2 Zusammenhang zwischen anamnestischer Mundtrockenheit und Speichelfließrate (nach V Dormenval et al. [6])

\begin{tabular}{|c|c|c|c|c|c|}
\hline \multirow[t]{2}{*}{$n=99$} & \multicolumn{2}{|c|}{ mit Symptomen } & \multicolumn{2}{|c|}{ ohne Symptome } & \multirow[t]{2}{*}{$P$} \\
\hline & $\%$ & $\mathrm{SSFR} / \mathrm{min}$ & $\%$ & $\mathrm{SSFR} / \mathrm{min}$ & \\
\hline $\begin{array}{l}\text { Regelmäßig trockener } \\
\text { Mund }\end{array}$ & 52 & 0,90 & 48 & 1,28 & 0,01 \\
\hline $\begin{array}{l}\text { Trockener Mund während } \\
\text { der Nacht }\end{array}$ & 43 & 0,80 & 57 & 1,24 & 0,01 \\
\hline $\begin{array}{l}\text { Trockener Mund während } \\
\text { des Tages }\end{array}$ & 40 & 0,70 & 60 & 1,30 & 0,0002 \\
\hline $\begin{array}{l}\text { Schwierigkeiten beim } \\
\text { Sprechen }\end{array}$ & 12 & 0,55 & 88 & 1,17 & 0,007 \\
\hline $\begin{array}{l}\text { Nächtliches Erwachen } \\
\text { um zu trinken }\end{array}$ & 34 & 0,81 & 66 & 1,24 & 0,03 \\
\hline $\begin{array}{l}\text { Mundtrockenheit er- } \\
\text { schwert das Tragen } \\
\text { einer Prothese }\end{array}$ & 21 & 0,30 & 79 & 1,00 & 0,03 \\
\hline $\begin{array}{l}\text { Schwierigkeiten zu Essen } \\
\text { und zu Schlucken }\end{array}$ & 15 & 0,52 & 85 & 1,17 & 0,002 \\
\hline
\end{tabular}

\section{Rekonstruktive Maßnahmen und Ernährung}

Es läge nahe anzunehmen, dass der Ersatz fehlender Zähne ausreichend sei um die Nahrungsaufnahme unserer Patienten $\mathrm{zu}$ verbessern, jedoch ist Ernährung nicht allein eine Frage der Kaufunktion. Gewohnheiten, Geschmack, kulturelle Gebräuche und nicht zuletzt finanzielle und organisatorische Aspekte bestimmen ebenfalls den Speiseplan. Darüber hinaus sind viele Patienten aufgrund systemischer Erkrankungen an eine spezielle Diät gebunden. Auch können Medikamente, die eine Mundtrockenheit verursachen, nicht immer durch Präparate ohne diese Nebenwirkung ersetzt werden. Die Mehrzahl der Studien zum Einfluss zahnärztlicher Intervention auf 
den Ernährungszustand konnten diesen daher nicht nachweisen $[10,12,28,36]$. Lediglich eine Arbeitsgruppe aus Montreal stellte 6 Monate nach Fixierung einer Unterkiefertotalprothese mittels Implantaten eine signifikante Verbesserung des Ernährungszustandes fest [27]. Es wird daher empfohlen, die Eingliederung neuer Prothesen mit einer Ernährungsberatung zu kombinieren [28]. Die Patienten sollten dazu angehalten werden mit dem neuen Zahnersatz auch den Verzehr anderer Nahrungsmittel auszuprobieren. Ferner sollte der Hinweis nicht fehlen, dass die $\mathrm{Zu}$ bereitung der Nahrung überdacht werden kann, da lange Garzeiten, die den Wert der Nährstoffe reduzieren, nicht mehr benötigt werden.

\section{Bedeutung der Kaufunktion}

Klassische Experimente in den 50ern zeigten, dass das Zerkauen von Nahrung keinen Einfluss auf deren Verdauung hat, jedoch führte Farrell seine Versuche an jungen, gesunden Probanden durch [7, 8]. Vergleichbare Untersuchungen an einem älteren Probandengut liegen leider nicht vor, so dass ein Zusammenhang zwischen dem Kauvorgang und der Verdauung für diese Population nicht vollständig ausgeschlossen werden kann. Sicher hingegen ist, dass eine gute Kaufunktion entscheidend zur (mundgesundheitsbezogenen) Lebensqualität beiträgt [16] Das problemlose Einnehmen von Mahlzeiten im Kreise der Familie oder im Restaurant tragen wesentlich zum psychosozialen Wohlbefinden bei [40] (Abb. 8). In gastlicher Gesellschaft und bei dekorativ gedecktem Tisch wird i.d.R. auch mehr gegessen [35]. Dahingegen fördert der Anblick einer durchgemixten Mahlzeit nicht gerade den Appetit.
Neurophysiologisch gehört die Kaufunktion zu einem der grundlegendsten Aktivitätsmuster. Im Hirnstamm wird der Central Pattern-Generator vermutet, der, ähnlich wie bei der Atmung, die rhythmischen Aktivitätsmuster initiiert und afferente Informationen aus der Mundhöhle umgehend integriert [3, 26]. Es wird vermutet, dass die Kaumuskulatur von beiden Hemisphären innerviert wird um trotz lokaler zerebraler Ausfälle die Kaufunktion sicherzustellen [18]. Sollte eine physiologisch derart abgesicherte und tief programmierte Funktion im Zeitalter des Foodmixers wirklich keinen biologischen Sinn mehr erfüllen? Eine Japanische Arbeitsgruppe konnte feststellen, dass die experimentelle Aufhebung der Kaufunktion durch Entfernen der Molaren bei alten Mäusen zur Verschlechterung des räumlichen Gedächtnisses sowie der Degeneration von Neuronen im Hippocampus führte [33]. Kann also Kauen das ZNS stimulieren und einer senilen Demenz vorbeugen? Sicherlich gibt es auf diesem Gebiet noch viel $\mathrm{zu}$ erforschen.

\section{Ausblick}

Die Ernährung der Patienten beschäftigt Zahnärzte, unabhängig welcher Altersgruppe diese angehören. Während bei Kindern und Jugendlichen vornehmlich das Kariesrisiko aufgrund kariogener Ernährung im Vordergrund steht, verursacht erbrochene Magensäure bei jungen Erwachsenen mit psychogenen Essstörungen oft palatinale Schmelzdefekte. Bei Senioren stehen hingegen der Zahnverlust und die damit verbundene eingeschränkte Kaueffizienz sowie die Mundtrockenheit und Dysphagie im Vordergrund. Die zahnärztliche Therapie kann bei Patienten mit
Abb. 8 Soziale Aktivitäten von 104 zahnlosen Patienten mit Totalprothesen sowie 16 Monate nach Eingliederung einer konventionellen Oberkiefer- und einer implantatgestützten Unterkieferprothese [Graphik gezeichnet nach Daten von Wismejer et al. 40]
Mit der Prothese, die ich derzeit trage, fühle ich mich wohl ...

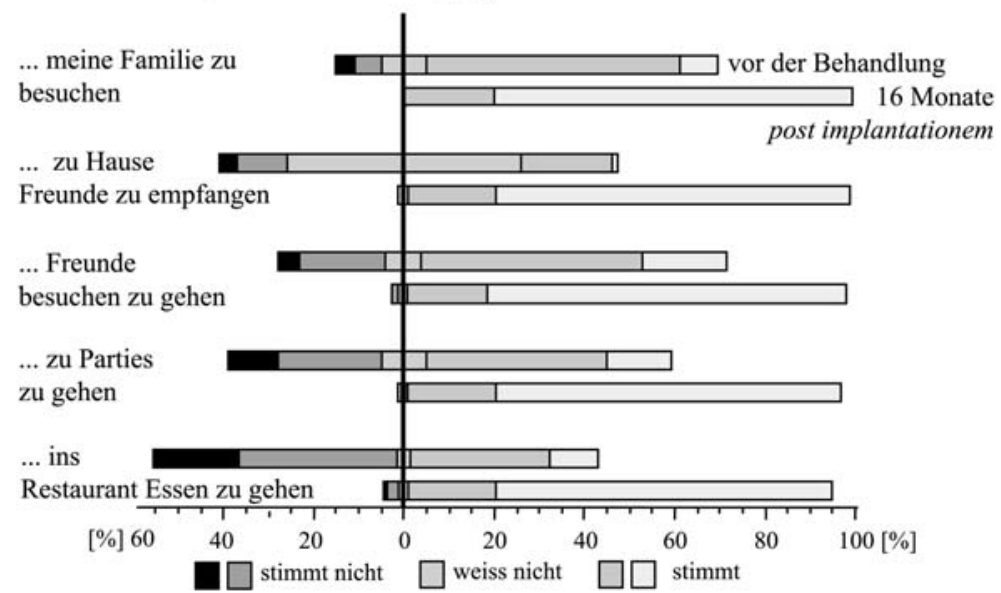


Unter- und Mangelernährung ein wichtiger Baustein im therapeutischen Mosaik sein.
Danksagung Die Autoren danken Prof. Robin Heath für die Überlassung der Abbildung der Siebe.

\section{Literatur}

1. Akeel R, Nilner M, Nilner K (1992) Masticatory efficiency in individuals with natural dentition. Swed Dent J 16:191-198

2. Boumendjel N, Herrmann F, Girod V, Sieber C, Rapin CH (2000) Refrigerator content and hospital admission in old people. Lancet 356:563

3. Dellow PG, Lund JP (1971) Evidence for central timing of rhythmical mastication. J Physiol 215:1-13

4. Dhaliwal J (2003) Does tooth loss affect ability to eat fruit and vegetables among British adults? MPhil thesis, University of Newcastle upon Tyne

5. Dormenval V, Budtz-Jørgensen $\mathrm{E}$, Mojon P, Bruyère A, Rapin CH (1995) Nutrition, general health status and oral health status in hospitalised elders. Gerodontology 12:73-80

6. Dormenval V, Budtz-Jørgensen, Mojon $\mathrm{Ph}$, Bruyère $\mathrm{A}$, Rapin $\mathrm{CH}$ (1998) Associations between malnutrition, poor general health and oral dryness in hospitalized elderly patients. Age and Ageing 27:123-128

7. Farrell JH (1956) The effect of mastication on the digestion of food. British Dental Journal 100:149-155

8. Farrell JH (1957) Partial dentures in the restoration of masticatory efficiency. Dent Pract and Dent Rec 7:375-379

9. Fontijn-Tekamp FA, Slagter AP, Van Der Bilt A, Van 'T Hof MA, Witter DJ, Kalk W, Jansen JA (2000) Biting and chewing in overdentures, full dentures, and natural dentitions. J Dent Res 79:1519-1524

10. Garrett NR, Perez P, Elbert C, Kapur KK (1996) Effects of improvements of poorly fitting dentures and new dentures on masticatory performance. J Prosthet Dent 75:269-275

11. Gunne HJ (1985a) Masticatory efficiency and dental state. A comparison between two methods. Acta Odont Scand 43:139-146

12. Gunne HJ (1985b) The effect of removable partial dentures on mastication and dietary intake. Acta Odontologica Scandinavica 43:262-278

13. Heath MR (1972) Dietary selection of elderly persons related to dental state. British Dental Journal 132:145-148

14. Heath MR (1982) The effect of maximum biting force and bone loss upon masticatory function and dietary selection of the elderly. Int Dent J 32:345-356
15. Ikebe K, Nokubi T, Sajima H, Kobayashi S, Hata $\mathrm{K}$, Ono $\mathrm{T}$, Ettinger RL (2001) Perception of dry mouth in a sample of community-dwelling older adults in Japan. Spec Care Dentist 21:52-59

16. Inglehart MR, Bagramian RA (2002) Oral Health-Related Quality of Life. Quintessenz, Chicago

17. Joshipura KJ, Willett WC, Douglass CW (1996) The impact of edentulousness on food and nutrient intake. J Am Dent Assoc 127:459-467

18. Kemppainen P, Waltimo A, Palomäki $\mathrm{H}$, Salonen $\mathrm{O}$, Könönen M, Kaste $\mathrm{M}$ (1999) Masticatory force and function in patients with hemispheric brain infarction and hemiplegia. J Dent Res 78:1810-1814

19. Krall E, Hayes C, Garcia R (1998) How dentition status and masticatory function affect nutrient intake. J Am Dent Assoc 129:1261-1269

20. Lenz E (1999) Zahnprothetischer Status bei den Senioren. In Micheelis W, Reich E (eds). Dritte Deutsche Mundgesundheitsstudie (DMS III) Deutscher Ärzteverlag, Köln S 385-411

21. Locker D (2003) Dental status, xerostomia and the oral health-related quality of life of an elderly institutionalized population. Spec Care Dentist 23:8693

22. Lucas PW, Luke DA (1983) Methods for analysing the breakdown of food in human mastication. Arch Oral Biol 28:813-819

23. Marshall Th, Warren J, Hand J, Xie X, Stumbo Ph (2002) Oral health, nutrient intake and dietary quality in the very old. J Am Dent Ass 133:1369-1379

24. Miyawaki S, Ohkochi N, Kawakami T, Sugimura M (2001) Changes in masticatory muscle activity according to food size in experimental human mastication. J Oral Rehabil 28:778-784

25. Millwood J, Heath MR (2000) Food choice by older people: the use of semi-structured interviews with open and closed questions. Gerodontology 17:25-32

26. Müller F, Heath MR, Ferman A, Davis $G$ (2002) Modulation of mastication during experimental loosening of complete dentures. Int J Prosthodontics 15:553-558
27. Morais JA, Heydecke G, Pawliuk J, Lund JP, Feine JS (2003) The effects of Mandibular Two-implant Overdentures on Nutrition in Elderly Edentulous Individuals. J Dent Res 82:53-58

28. Moynihan PJ, Butler TJ, Thomason JM, Jepson NJA (2000) Nutrient intake in partially dentate patients: the effect of prosthetic rehabilitation. J Dent 28:557-563

29. Mowlana F, Heath MR, Van der Bilt A, Van der Glas HW (1994) Assessment of chewing efficiency: a comparison of particle size distribution determined using optical scanning and sieving of almonds. J Oral Rehabil 21:545-551

30. Newton JP, Yemm R, Abel RW, Menhinick S (1993) Changes in human jaw muscles with age and dental state. Gerodontology 10:16-22

31. Nitschke I, Hopfenmüller W (1996) Die zahnmedizinische Versorgung älterer Menschen. In Mayer KU, Baltes PB (eds) Die Berliner Altersstudie Akademieverlag. Berlin S 429-451

32. Nitschke I, Müller F, Hopfenmüller W (2001) The uptake of dental services by elderly Germans. Gerodontology 18:114-120

33. Onozuka M, Watanabe $\mathrm{K}$, Mirbod SM, Ozono S, Nishiyama K, Karasawa N, Nagatsu I (1999) Reduced mastication stimulates impairment of spatial memory and degeneration of hippocampal neurons in aged SAMP8 mice. Brain Res 826:148-153

34. Prinz JF (1999) Quantitative evaluation of the effect of bolus size and number of chewing strokes on the intra-oral mixing of a two-colour chewing gum. J Oral Rehabil 26:243-247

35. Rapin CH (2004) Stratégies pour une vieillesse réussie. Medecine $\&$ Hygine, Geneva

36. Roumanas E et al (2002) A randomized clinical trial comparing the efficacy of mandibular implant-supported overdentures and conventional dentures in diabetic patients. Part V Food Preference comparisons. J Prosthet Dent 87:62-73

37. Sheiham A, Steele JG, Marcenes W, Lowe C, Finch S, Bates CJ, Prentice A, Walls AWG (2001 a) The Relationship among Dental Status, Nutrient intake and Nutritional Status in Older People. J Dent Res 80:408-413 
38. Sheiham A, Steele J (2001 b) Does the condition of the mouth and teeth affect the ability to eat certain foods, nutrient and dietary intake and nutritional status amongst older people? Public Health Nutr 4:797-803
39. Walls AWG, Steele JG (2004) The relationship between oral health and nutrition in older people. Mechanisms of Ageing and Development 125:853-857

40. Wismejer D, Van Waas MAJ, Vermeeren JIJF, Mulder J, Kalk W (1997) Patient satisfaction with implant-supported mandibular overdentures. Int J Oral Maxillofac Surg 26:263-267
41. Wöstmann B, Wickop H, Kolb G, Ferger P (1997) Zahnärztlich geriatrisches Assessment zur objektiven Einschätzung der zahnärztlich prothetischen Versorgung und des Ernährungszustandes älterer Patienten. Geriat Forsch 7:112-113 